\title{
The effects and effectiveness of being trained in Transactional Analysis. An empirical study
}

\author{
(C) 2012 Rosa C. Nowak
}

\begin{abstract}
An empirical qualitative study on the effectiveness of non-therapeutic TA-training is presented. It was carried out during the period 2008-2010 within the frame of a PhD thesis at Sigmund Freud University in Vienna. The results of this study have been published under the title of "Transaktionsanalyse und Salutogenese" (Waxmann 2011). The present article presents and discusses the research questions, the selection of the study method, the research design and some specific results of the study, such as changes in the Sense of Coherence.
\end{abstract}

\section{Introduction}

How effective and useful is Transactional Analysis? What changes does it generate in the life of those who deal with TA? How can those who undergo TA training in non-therapeutic contexts, that is, in the social, pedagogical and organizational realms benefit from it? These questions were the starting point of an empirical study carried out in the scope of a PhD thesis at Sigmund Freud University in Vienna during the years $2008-2010$.

Previous studies have already shown that TA-trainings can be of great benefit and they can broaden professional competences (e.g. Beck-Neumann and Huschens 2007). The questions that still needed to be addressed are whether TA-training also affects personal well-being beyond the improvement of professional training; whether it improves the emotional quality of life in any way, and whether it is possible to identify changes generated by TA-training in private life, in the relationship to partners, family and friends.

The second theory that underlies this study is Antonovsky's concept of salutogenesis (1979 and 1987/1997). Being 13 years younger than Eric Berne, Antonovsky produced several publications, especially during the 70 s and $80 \mathrm{~s}$. He designed a comprehensive model based on stress research and on medicalsociological considerations to explain the origin of health and disease. There is no space here to explain this model in detail, but a compact explanation is provided elsewhere (cf. Nowak 2011, pages 77-91).
The fundamental questions that Antonovsky intends to answer are as follows: How do human beings keep their balance within the turbulences of everyday life? How can they keep their equilibrium beyond the numerous stressors that surround them? What factors contribute to well-being? A core aspect in this theory is the concept of Sense of Coherence or SOC, according to which individuals are likely to be healthy and enjoy well-being (1.) if they understand what happens in their environment, (2.) if they can cope with everyday life challenges and (3.) if they can make sense out of their daily activities. Thus, according to Antonovsky, the stronger the three components of Sense of Coherence are developed, namely comprehensibility, manageability and meaningfulness, the more an individual can experience a strong feeling of confidence, trust and well-being. Numerous empirical studies have validated this hypothesis. However, the influence of SOC on physical aspects of health is not as clear yet as was assumed by Antonovsky. On the other hand, the relationship between psychological condition, emotional well-being, self-estimated health and the SOC components is more evident.

The purpose of the present study is to link the exploration of TA-effectiveness with the concept of sense of coherence and to address the question whether SOC changes and even improves by learning and applying the concept of TA. Furthermore, alongside this special focus, we also want to find out in a broader sense what kind of changes in (private) everyday life in general people are aware of as they intensively deal with TA. The theory of salutogenesis has an additional function in this study: it constitutes the basis for the interpretation of the collected empirical data. That is, the collected data is interpreted in the light of Antonovsky's model.

As a result of an extensive consideration of pros and cons of quantitative and qualitative research, we opted for a qualitative approach (Lamnek 2005), which seemed to be the most adequate method to deal with the present issue. One of the advantages of qualitative research is that semi-structured qualitative interviews as the ones we carried out - allow not only to get answers to the topics and questions posed by the 
researcher, for example by means of a questionnaire, but they also give enough free way for interviewees to address any topic they may consider relevant. Thus, new issues that have not been addressed by the researcher are spontaneously brought up. Besides, a qualitative method makes it possible to go beyond the collection of isolated factors and detect deep interconnections and interrelationships.

Qualitative interviews normally begin with specific introductory questions, as for example in this case: "How long have you been interested in TA? or "What made you choose TA?", "What changes have you noticed?" Although such questions are to be answered concretely, they are also there to stimulate the conversation, to keep up a meaningful dialogue and to encourage interviewees to talk about their personal lives. As a result, very lively stories, vivid reports, pointed quotations and interesting thoughts come up in the course of the interview, providing insight into everyday activities as well as thoughts and feelings of persons that have undergone TA-training.

A total of 12 persons participated in interviews that lasted in part for several hours. The inclusion criteria were defined as follows: Interviewees should live in Austria and they should have completed at least threeyear TA training. The only exclusion criteria were personal friendship or professional co-operation with the interviewer. As is usual in qualitative research, subjects were otherwise selected according to heterogeneity of characteristics with respect to age, profession and place of residence. Two thirds were women, one third men; only about one third of the subjects aimed at a higher qualification in TA or had already acquired one. TA-training was in part made in Germany or with Swiss trainers. All interviews were completely transcribed; the resulting text of 326 pages was then analysed and interpreted in detail (Mayring 2010). Rather than mainly distributing answers and assertions in numbers and percentages, this kind of text analysis categorizes contents and looks for diverse patterns of thought, feeling and action that can be unveiled in relation to the research issue. Results are presented in order to show diversity in life (as can be seen in the reports), to compare different statements as such revealing possible contrasts instead of harmonizing them artificially.

In spite of this open approach and despite the divergent opinions expressed by interviewees, partly about TA in general and partly as appraisal of some TA-trainers, there is absolutely common consensus amongst interviewees that TA models are useful and valuable instruments for everyday life. They are considered to be a support for better understanding even highly difficult relationships and communication situations. TA concepts make it possible to identify underlying structures and core aspects that help to understand what happens in one's own psyche and in interpersonal contacts. As a consequence of this awareness process, individuals are able to cope better with themselves and with communication situations, to open up new scopes of action, to be more purposefully active and not to surrender to an unknown fate. It is interesting to note that "to understand something and then be able to cope with it better" is a persistent statement throughout the interviews and constantly appears, almost as a set expression. This means that insight and realization are always intimately related with consequent actual (modified, improved) actions and ways of behaving. Associations of this sort do not always take place in psychological theories and in everyday life. Numerous citations, descriptions and concrete reports from the interviewees' accounts show this growing awareness and improved capacity to act, a fact that clearly corresponds to the first two components of Sense of Coherence, namely "comprehensibility" and "manageability". Obviously, an improvement of these two factors does not necessarily imply that absolutely all incidents in life will now be understood and successfully mastered. Yet Antonovsky defined four areas that, in his view, are of crucial significance for every human being, are essential for a balanced SOC, and should be understood and wisely managed: (one's own) feelings, personal relationships, basic activities, and existential questions (amongst which Antonovsky includes problem solving). To the question as to the contexts in which there was an improvement in understanding and knowing how to handle situations, interviewees spontaneously mentioned one or several of these four contexts defined by Antonovsky. This is especially striking, considering that interviewees were not directly asked about possible empowerment of the SOC (or of its components) for two obvious reasons: on the one hand it was not possible to assume that all participants were familiarized with Antonovsky's model; on the other hand, the intention was to perform the interview in an objective way, leaving free way to different facts and assertions, without providing pre-determined answers. In order to assure this sort of neutrality and objectivity in the collection of data, interviewees were simply asked about changes they had observed that were associated in one way or another with their dealing with TA. It was only during the analysis of the transcribed interview texts that it became obvious that many of the statements corresponded exactly to Antonovsky's definition of the SOC factors and to their stabilization and improvement. In order to give an accurate account of the statements in the texts related to SOC, a template was designed for this analysis (cf. Nowak 2011, p. 113), which connects the three components of Sense of Coherence with the four above-mentioned areas of life. This analysis scheme makes it possible to accurately and contextually identify statements on comprehensibility, manageability, meaningfulness.

According to the interviewees' report, the factor "meaningfulness", the rather emotional factor of experiencing sense in daily life is also improved, although this is not so pronounced and frequent as the empowerment of the other two factors that affect behaviour and, first and foremost, cognitive understanding. A cross connection between TA and the concept of SOC is also possible due to the fact that the three SOC factors are characterized by being cognitive, behaviour-oriented and emotional, which corresponds 
to the typical associations made in TA of thought, feelings and behaviour. This study does confirm a clear empowerment of all three SOC components (in different degrees).

In this respect the interviewees also reported to a certain extent an improved general emotional state and an enhanced well-being in general, which they explain as consequence of being able to put things in perspective, to understand incidences and to consciously choose appropriate behaviour patterns. But the real fundamental change and improvement of wellbeing through dealing with TA indicated by most interviewees was due to a completely different factor: having learned and practiced the okay-position. This position of respect, esteem and acknowledgement to oneself and to others leads even more to a basic sense of safety and confidence, which increasingly influences everyday life. Thus, practising the plus-plus position improves the emotional quality of life in a way that has proven to go deeper than cognitive awareness or new management options. Thus, from a theoretical point of view, the plus-plus-position could actually be incorporated into the concept of $\mathrm{SOC}$ and could as such be the basis of a positive attitude towards life. Plus-plus is not only "good" in an ethical sense; it also feels good, both for those who practice it and for their fellowmen. Ethics and emotional well-being are linked together in the plus-plus position, both in theory and practice, and do not stand in contradiction.

A further result of this study that can be briefly referred to here, concerns the interviewee's motivation to undergo TA-training. It is not only the professional background and the motivation to broaden professional skills what plays an important role here. Surprisingly enough, most interviewees indicated personal and private reasons rather than professional goals as their primary motivation to join a training course. Some of them report having exclusively a private motive, such as the need to cope with divorce or the urge to improve communication with the partner and even grieving after the loss of a parent. It is often the case that the actual reason to get TA-training is a critical incident or phase of life combined with professional motives.

Pure professional motivations are in this study the exception. The decision to focus on TA and not on any other psychological theory is in many cases motivated by a first contact with this approach at university, where it is mainly taught in relation to economic studies rather that with psychology. Often, this brief contact with TA dates back many years but it was apparently so influential that it led to opting for TA-training.

Concerning the private life of the interviewees, they extensively explained the way TA was useful for them, how their family, children and partners reacted to the situation that had changed due to TA and the kind of influence exerted by TA in partner relationships in which one of the partners is not interested in TA or in any other psychological theory. Thus, this issue plays a crucial role in the interpretation of the research results (Nowak 2011, pp 169-197). There is not enough space here to go into detail on the different life situations; besides, it would not make much sense to present synthesized partial results, since qualitative studies are characterized by the plasticity of detailed reports, by the vividness of results and personal reports, in which apparently unimportant aspects become in fact meaningful aspects in the description; not least because the complexity of results can only be accounted for when presented in detail.

This is also valid for other issues that were brought up in the interviews: the different ways and methods in which in each single case TA- concepts were integrated in everyday life; the special way of dealing with preferential TA-models; the search for one or several preferred concepts, the interviewee's relation to world view, ethics and spirituality in the context of TA, and last but not least - the degree of satisfaction of trainees with their completed training and the question as to what factors they considered to have mainly contributed to success (or lack of success) in their training. All these aspects are given at length (140 pages) in the publication of this research as results, in terms of the results of text analysis and interpretation (Nowak 2011, pp 148-289). Our study also sought to detect those special factors of Transactional Analysis that have rendered in part surprising results in their application. Thus, this study provides information not only for practising transactional analysts but also for readers who are not familiar with TA and are possibly considering TA-training. In this way they can get a picture of what it actually means to "apply TA". The possible empowerment of Sense of Coherence is just one of many aspects, yet it is essential if one considers for example empirical studies that, along with physical well-being, even confirm that there is a relationship between a strong SOC and the human immune system (Lutgendorf u.a. 1999).

So, what is the meaning and contribution of the results of this study? First of all it provides an analysis of successful training processes and their results. Interviewees are in most cases persons with a positive attitude towards TA even long after completion of training and who have had positive experiences and maintain contact with Transactional Analysis in one way or another, even if it "just" means to put the plus-plus position into practice, without having any sort of contact with other transactional analysts. What cannot be derived from this study, however, is the frequency of the collected results in terms of quantities and percentages with reference to all TA-trainees, since this is not intended to be a quantitative study. In principle, qualitative studies are not numerically oriented; still, qualitative research can lead to generalizations in a different sense, a sense that goes beyond their significance for twelve interviewees. Even if the purpose is to represent individual experiences, it cannot be assumed that such studies are exclusively about individual cases that have nothing in common with other people. What can be generalized is a set of patterns of thought, action and experience that can somehow reoccur both in one-self and in others. This is in essence only one special instance of the fact that people are unique in their individuality, but at the same 
time they share common features with other people. The second aspect that allows drawing generalizations from results are possibilities that can arise in principle: What was a possibility in particular cases is, from a logical point of view, basically no impossibility. The individual training processes and the achieved successful results cannot be transferred one to one to other people and their situation of life, but they may represent a possible trend in development that may be open to others, at least in part or to some extent.

Frustrated TA trainees who give up their training or who reject TA theory in general are included in this study but they actually represent a minority. Amongst the reasons reported for a negative attitude towards TA are conflicts within the group or trainees. It would be certainly interesting and necessary to carry out an additional study on frustrated TA trainees and to find out other reasons for a subjectively experienced failure or loss of contact.

One of the conclusions that can be drawn from the reports, which are mainly positive, is the fact that TAtraining not only broadens competencies but it also enhances personal awareness of whole-being and oneness with oneself, which is otherwise achieved rather with psychotherapeutic efforts (Fäh and Fischer 1998). The changeability of Sense of Coherence by means of TA is not only information of interest for TAtrainers, this being one of the aspects of its effectiveness, but it can also be of relevance for people who consider being trained in TA.

The results of the study suggest that the presence of Transactional Analysis in universities is not to be underestimated, when it comes to the dissemination of this approach. Some interviewees expressed that they would probably not have considered TA-training if they had not had this marked experience during their university studies. A broader inclusion of TA in universities would naturally imply more research in this area, and at the same time it would increase its dissemination and consequently awaken the interest in TA in potential candidates. Joint efforts between institutions that focus on TA-training and university TAresearch would be highly recommendable. It would take account of the fact that training needs research to the same extent to which research could not do without the praxis of TA-application.

\section{References}

Antonovsky, Aaron 1979: Health, Stress and Coping. New perspectives on mental and physical well-being. San Francisco: Jossey-Bass Publishers.

Antonovsky, Aaron 1987/1997: Salutogenese. Zur Entmystifizierung der Gesundheit (Dt. erw. Hrsg. von Alexa Franke). Tübingen: Dgvt-Verlag [Unraveling the Mystery of Health. How People Manage Stress and Stay Well. San Francisco: Jossey-Bass Publishers].

Beck-Neumann, Gundel; Huschens, Anne 2007: Wie Transaktionsanalyse wirkt. Ergebnisse der Befragung im Rahmen des Selbstevaluationsprojekts 2005/ 2006. In: ZTA 24 (3), S. 219-247.

Lamnek, Siegfried 2005: Qualitative Sozialforschung. Lehrbuch. 4. Aufl. Weinheim: Beltz.

Lutgendorf, Susan K. u.a. 1999: Sense of Coherence Moderates the Relationship Between Life Stress and Natural Killer Cell Activity in Healthy Older Adults. In: Psychology and Aging 14 (4), S. 552-563.

Mayring, Philipp 2010: Qualitative Inhaltsanalyse. Grundlagen und Techniken. 11. Aufl. Weinheim: Beltz.

Nowak, Rosa C. 2011: Transaktionsanalyse und Salutogenese. Der Einfluss transaktionsanalytischer Bildung auf Wohlbefinden und emotionale Lebensqualität. Psychotherapiewissenschaft in Forschung, Profession und Kultur. Bd. 2, hg. v. Bernd Rieken. Münster, New York: Waxmann. 